\title{
ON THE INTERIOR OF SUBSEMIGROUPS OF LIE GROUPS
}

\author{
KARL H. HOFMANN AND WOLFGANG A. F. RUPPERT
}

\begin{abstract}
Let $G$ denote a Lie group with Lie algebra $\mathfrak{g}$ and with a subsemigroup $S$ whose infinitesimal generators generate $\mathfrak{g}$. We construct real analytic curves $\gamma: \mathbf{R}^{+} \rightarrow S$ such that $\dot{\gamma}(0)$ is a preassigned tangent vector of $S$ at the origin and that $\gamma(t)$ is in the interior of $S$ for all positive $t$. Among the consequences, we find that the boundary of $S$ has to be reasonably well behaved. Our procedure involves the construction of certain linear generating sets from a given Lie algebra generating set, and this may be of independent interest.
\end{abstract}

\section{INTRODUCTION}

This paper presents results on subsemigroups of Lie groups whose geometric significance is best explained in terms of control theory.

Let $E$ be a system of smooth vector fields, defined on a manifold $M$-the space of states. Then to every $X \in E$ and each point $m \in M$, there exists a unique trajectory $t \mapsto x_{m}(t)$ satisfying $\dot{x}_{m}(t)=X\left(x_{m}(t)\right)$ and $x_{m}(0)=m$. Moreover, for each time $t$ the map $m \mapsto x_{m}(t)$ is a diffeomorphism; we denote this diffeomorphism by $\exp t \cdot X$, so that $x_{m}(t)=(\exp t \cdot X)(m)$. As $X$ ranges through the generating set $E$ and $t$ through $\mathbf{R}^{+}=[0, \infty[$, the maps $\exp (t \cdot X)$ generate a semigroup $S$ of diffeomorphisms and, together with their inverses, a group $G$; in many cases of interest $G$ is a Lie group. Steering this system means that we are allowed to choose a function $t \mapsto X_{t}:[0, T] \rightarrow \mathbf{R}^{+} \cdot E$ from a set of previously given steering functions and to obtain for every point $m \in M$ a trajectory $t \mapsto y_{m}(t)$ starting at $y(0)=m$ and satisfying $\dot{y}_{m}(t)=X_{t}\left(y_{m}(t)\right)$. If our steering functions are the piecewise constant maps $[0, T] \rightarrow E,\left(T \in \mathbf{R}^{+}\right)$, then every point $p$ on a trajectory $y_{m}$ can be written as

$p=y_{m}(t)=\left(\exp \left(t-t_{n-1}\right) \cdot X_{n}\right) \circ\left(\exp \left(t_{n-1}-t_{n-2}\right) \cdot X_{n-1}\right) \circ \cdots \circ\left(\exp t_{1} \cdot X\right)(m)$,

hence these points exactly form the orbit $S m$ of the semigroup $S$. With a more liberal supply of steering functions, we reach the points of $\overline{S m}$.

The geometry of this set of reachable points is of great interest in geometric control theory. Does it have interior points? If so, how singular can boundary

Received by the editors January 3, 1989 and, in revised form, February 23, 1989.

1980 Mathematics Subject Classification (1985 Revision). Primary 22E15, 22E60; Secondary $22 \mathrm{~A} 15$.

Key words and phrases. Lie group, infinitesimally generated semigroup, analytic curve, analytic deformation, boundary. 
points be? Can one reach its interior starting from $m$ on a smooth curve? Clearly, all of these questions remain relevant in the particular case where $M=$ $G$ and $G$ acts on $M$ by left translations. Answers to our questions in this special case promise answers in the general one.

In the special case the problem can be posed in a slightly more general and concise manner:

Let $E$ be a subset of the Lie algebra $\mathfrak{g}$ of a Lie group $G$ and suppose that $S$ is a subsemigroup of $G$ with $\exp \mathbf{R}^{+} \cdot E \subseteq S$.

(i) Does $S$ have nonempty interior int $S$ in $G$ ?

(ii) Are there smooth curves in $\bar{S}$ connecting 1 with points in int $S$ ?

It is known that int $S \neq \varnothing$ as soon as the Lie algebra generated by $E$ in $\mathfrak{g}$ is all of $\mathfrak{g}$. Better still, the interior is a dense ideal of $S$ and agrees with int $\bar{S}$. (Cf. [1, Chapter V] for more information and references.) Our result will reestablish this insight and will add information allowing us to answer the question on arc connectivity in the affirmative. Indeed we shall show that, under the assumption that $E$ generates $\mathfrak{g}$ as a Lie algebra, to each point $p \in \bar{S}$ (notably, to $\mathbf{1}$ or any boundary point) there is a real analytic curve $\gamma: \mathbf{R}^{+} \rightarrow \bar{S}$ with $\gamma(0)=p$ and $\gamma(t) \in$ int $S$ if $t>0$. In fact, there are generalized one-parameter subgroups, that is, curves of the form $t \mapsto \gamma(t)=\exp t \cdot X_{1} \cdots \exp t \cdot X_{n}$ with suitable elements $X_{j} \in \mathbf{R}^{+} \cdot E$ such that $\gamma(t) \in \operatorname{int} S$ for $0<t \leq 1$.

A subtangent vector $X$ of $S \subseteq G$ at 1 is an element $X \in \mathfrak{g}$ satisfying $X=\lim r_{n} \cdot X_{n}$ with $\exp X_{n} \in S$ and $X_{n} \rightarrow 0$. The set of all subtangent vectors of $S$ at 1 is denoted $\mathrm{L}(S)$ (cf. [1]).

It is obvious that the initial velocity $\dot{\gamma}(0)$ of $\gamma$ is a subtangent vector of $S$ at 1 ; once one such curve $\gamma$ is obtained, it is not hard to show that every subtangent vector of $S$ at $\mathbf{1}$ is the velocity vector of an analytic curve diving into the interior int $S$. Also we establish quickly that every connected component of $\bar{S}$ is connected by smooth arcs.

These results call for some comment. Firstly, even in vector groups like $\mathbf{R}^{2}$, for which we can write $\mathfrak{g}=G$ with the identity function as exponential function, subsemigroups can have rather bizarre boundaries. The next best thing in semigroups to the famous "closure of the $\sin \frac{1}{x}$ graph" is the following example ("the Shark's Tooth").

Example 0.1. For each $n \in \mathbf{N}$ define $S_{n} \stackrel{\text { def }}{=}\left\{(x, y) \in \mathbf{R}^{2} \mid 2^{-n}<2^{n} x<y\right\}$. A straightforward calculation shows that $S_{m}+S_{n} \subseteq S_{m}$ whenever $m<n$, hence $S \stackrel{\text { def }}{=} \bigcup_{n \in \mathbf{N}} S_{n}$ is an open subsemigroup of $G$. Also, $\mathbf{1}=(0,0) \in \bar{S}$.

This construction yields a connected closed submonoid $\bar{S}$ of $\mathbf{R}^{2}$ with dense interior such that the path component of the identity $\mathbf{1}$ is $\{0\} \times \mathbf{R}^{+}$and agrees with $\mathbf{L}(S)=\exp \mathbf{L}(S)$. No arc whatsoever starting from a boundary point of the form $(0, y)$ can ever reach the interior int $S$. (This example also plays a role in the theory of congruences on open subsemigroups of Lie groups, cf. [2] or $[1, \S \mathrm{V} .7]$.) 
Example 0.1 shows how badly arc connectivity can fail for connected closed monoids with dense interior if they are far enough from being "infinitesimally generated" (see [1]) and emphasizes the importance of the hypothesis that $E$ generates $\mathfrak{g}$ as Lie algebra. We know that if $S$ is an open semigroup in $G$ with $\mathbf{1} \in \bar{S}$ then $S$, and therefore $\bar{S}$, too, must be connected if $G$ is connected and solvable (cf. [3]); however, this does not imply that $\bar{S}$ is pathwise connected.

It is also instructive to recall at this point that any semisimple Lie algebra can be generated by two vectors. Thus semigroups of arbitrarily large dimension may be generated by $t w o$ one-parameter semigroups. An intuitively persuasive low dimensional illustration of this phenomenon is provided by the smallest nilpotent nonabelian Lie algebra and its simply connected Lie group. It contains a semigroup, sometimes called "the Heisenberg beak" which is generated by $\exp \mathbf{R} \cdot E$, where $E$ consists of two vectors.

Example 0.2. Denote with $G$ the Heisenberg group, written as the set of all real 3-vectors with multiplication

$$
(a, b, c)\left(a^{\prime}, b^{\prime}, c^{\prime}\right)=\left(a+a^{\prime}, b+b^{\prime}, c+a b^{\prime}+c^{\prime}\right)
$$

and define $S$ to be the open subsemigroup

$$
S=\{(a, b, c) \in G \mid 0<a, 0<b, 0<c<a b\} .
$$

Here $\mathbf{L}(S)$ is a two-dimensional wedge and generates the whole Lie algebra $\mathfrak{g}$. Every two points $(a, b, c)$ and $\left(a^{\prime}, b^{\prime}, c^{\prime}\right)$ in $\bar{S}$ can be joined by an analytic arc $\alpha:[0,1] \rightarrow \bar{S}$ with $\alpha(] 0,1[) \subset S$. For instance we may define $\alpha(t)=\left(t a+(1-t) a^{\prime}+t(1-t) \varepsilon, t b+(1-t) b^{\prime}+t(1-t) \varepsilon, t^{2} c+(1-t)^{2} c^{\prime}\right)$, where $\varepsilon$ is any positive real number. However every generalized one-parameter semigroup

$$
\gamma: \mathbf{R}^{+} \rightarrow \bar{S}, \quad t \mapsto \exp t \cdot X_{1} \exp t \cdot X_{2} \cdots \exp t \cdot X_{n}
$$

meeting the boundary of $S$ in a point $\neq 1$ must remain on the surface without ever meeting the interior of $S$.

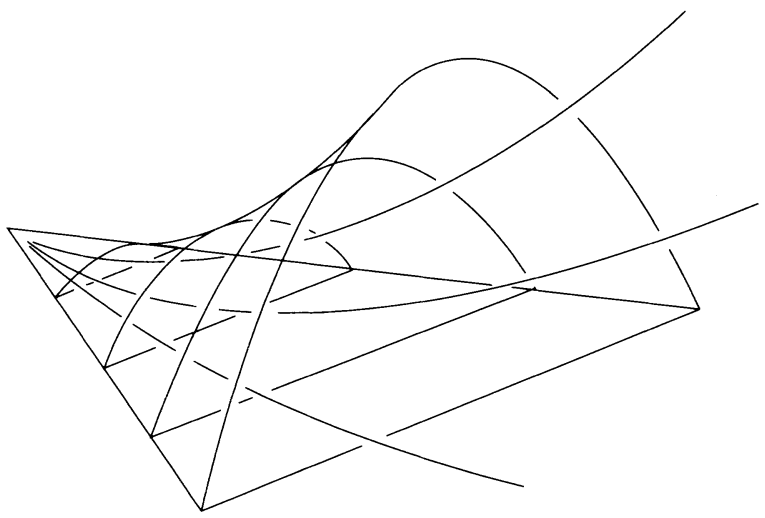




\section{Generators of A ReAl Lie algebra}

The first difficulty encountered in the proof of our main result is that there is no obvious way of utilizing the assumption " $E$ generates $\mathfrak{g}$ as a Lie algebra." What we need is a machinery associating with every set $E$ of Lie algebra generators a set $B$ which linearly spans $\mathfrak{g}$ and is structured suitably for our purposes. Such a device is provided by the following result, which may also be of independent interest.

Theorem 1.1. Suppose that the set $E \subset \mathfrak{g}$ generates $\mathfrak{g}$ as a Lie algebra and satisfies $\mathbf{R}^{+} \cdot E=E$. Then there exists an ordered $n$-tuple $\left(X_{1}, \ldots, X_{n}\right) \in E^{n}$ such that for every $t \in] 0,1]$ the space $\mathfrak{g}$ is the linear span of

$$
\left\{X_{n}, e^{t \cdot \operatorname{ad} X_{n}} X_{n-1}, \ldots, e^{t \cdot \operatorname{ad} X_{n}} \ldots e^{t \cdot \operatorname{ad} X_{2}} X_{1}\right\} .
$$

The proof of this theorem will proceed through several steps.

For the purposes of the proof only, we first introduce the following ad hoc terminology.

Definition 1.2. (i) For any choice of elements $X_{1}, \ldots, X_{n} \in E$, we shall call the set

$$
B\left(X_{1}, \ldots, X_{n}\right) \stackrel{\text { def }}{=}\left\{X_{n}, e^{\text {ad } X_{n}} X_{n-1}, \ldots, e^{\text {ad } X_{n}} \ldots e^{\text {ad } X_{2}} X_{1}\right\}
$$

a basic system.

(ii) A vector subspace $\mathfrak{v}$ of $\mathfrak{g}$ shall be called nice if it is spanned by a basic system $B\left(X_{1}, \ldots, X_{n}\right)$ and has maximal dimension among all vector spaces spanned by basic systems.

Lemma 1.3. If $\mathfrak{v}=\operatorname{span} B\left(X_{1}, \ldots, X_{n}\right)$ is nice, then $E \subseteq \mathfrak{v}$.

Proof. Suppose there is an $X \in E$ with $X \notin \mathfrak{v}$. Then $e^{-\mathrm{ad} X} X=X \notin \mathfrak{v}$, and thus $X \notin e^{\operatorname{ad} X} \mathfrak{v}$. Now let $\mathfrak{v}^{\prime}=\mathbf{R} \cdot X+e^{\operatorname{ad} X} \mathfrak{v}=\operatorname{span} B\left(X, X_{1}, \ldots, X_{n}\right)$. Then $\operatorname{dim} \mathfrak{v}^{\prime}=\operatorname{dim} \mathfrak{v}+1$ and $\mathfrak{v}^{\prime}$ is spanned by a basic system. This contradicts the maximality of the dimension of $\mathfrak{v}$.

Lemma 1.4. If $\mathfrak{v}=\operatorname{span} B\left(X_{1}, \ldots, X_{n}\right)$ is nice and $X \in E$, then $e^{\operatorname{ad} X} \mathfrak{v}$ is nice. Proof. We consider $\mathfrak{v}^{\prime}=\mathbf{R} \cdot X+e^{\text {ad } X} \mathfrak{v}=\operatorname{span} B\left(X, X_{1}, \ldots, X_{n}\right)$. Then $\operatorname{dim} \mathfrak{v}^{\prime} \geq \operatorname{dim} \mathfrak{v}$ and $\mathfrak{v}^{\prime}$ is spanned by a basic system. Hence $\mathfrak{v}^{\prime}$ is nice. By Lemma 1.3 we know that $X \in \mathfrak{v}$, whence $\mathfrak{v}^{\prime}=e^{\text {ad } X} \mathfrak{v}$.

Lemma 1.5. There exist basic systems which span $\mathfrak{g}$.

Proof. It suffices to find $X_{1}, \ldots, X_{n} \in E$ such that $B\left(t \cdot X_{1}, \ldots, t \cdot X_{n}\right)$ spans the Lie algebra of $G$ for all $t \in] 0,1]$. We form

$$
\mathfrak{u}=\bigcap\{\mathfrak{v} \mid \mathfrak{v} \text { is nice }\} .
$$

Then $E \subseteq \mathfrak{u}$ by Lemma 1.3, and $\mathfrak{u}$ is invariant under $e^{\text {ad } X}$ for every $X \in E$ by Lemma 1.4. It follows that $e^{t^{\bullet} \cdot \mathrm{ad} X} \mathfrak{u} \subseteq \mathcal{u}$ for $X \in E$ and $t \in \mathbf{R}$, and thus 
$[X, Y]=\lim _{0 \neq t \rightarrow 0} t^{-1}\left(e^{t \operatorname{ad} X} Y-Y\right) \in \mathfrak{u}$ for any $Y \in \mathfrak{u}$. Now the normalizer $\{Z \in \mathfrak{g} \mid[Z, \mathfrak{u}] \subseteq \mathfrak{u}\}$ of $\mathfrak{u}$ in $\mathfrak{g}$ contains $E$, hence the Lie algebra generated by $E$. Thus $\mathfrak{u}$ is an ideal in $\mathfrak{g}$, and since it contains $E$ it agrees with $\mathfrak{g}$. Therefore every basic system $B\left(X_{1}, \ldots, X_{n}\right)$ spanning a space of maximal dimension spans $\mathfrak{g}$.

Proof of Theorem 1.1. We begin with a basic system $B\left(X_{1}, \ldots, X_{n}\right)$ spanning $\mathfrak{g}$ according to Lemma 1.5 .

Let $m$ denote $\operatorname{dim} \mathfrak{g}$ and fix a basis of $\mathfrak{g}$. Then with respect to this basis, the coefficients of the vectors in the basic system

$$
B\left(t \cdot X_{1}, \ldots, t \cdot X_{n}\right)
$$

form an $m \times n$-matrix giving us $\left(\begin{array}{c}n \\ m\end{array}\right)$ subdeterminants of size $m \times m$, say

$$
D_{1}(t), \ldots, D_{\left(\begin{array}{c}
n \\
m
\end{array}\right)}(t) \text {. }
$$

Since $\mathfrak{g}=\operatorname{span} B\left(X_{1}, \ldots, X_{n}\right)$, there is a $k \in\left\{1, \ldots,\left(\begin{array}{c}n \\ m\end{array}\right)\right\}$ such that $D_{k}(1) \neq$ 0 . The zeroes of the nonconstant real analytic function $D_{k}$ form a discrete subset of $\mathbf{R}$, so there exists an $\varepsilon>0$ such that $0<t \leq \varepsilon$ implies $D_{k}(t) \neq 0$. Now $\operatorname{span} B\left(t \cdot X_{1}, \ldots, t \cdot X_{n}\right)=\mathfrak{g}$ for all $\left.\left.t \in\right] 0, \varepsilon\right]$ and replacing the set $\left\{X_{1}, \ldots, X_{n}\right\}$ by $\left\{\varepsilon \cdot X_{1}, \ldots, \varepsilon \cdot X_{n}\right\}$ we get the results claimed.

The proof of Theorem 1.1 actually yields a slightly sharper result:

Corollary 1.6. Under the circumstances of Theorem 1.1, there exist basic systems spanning $\mathfrak{g}$, and if $B\left(X_{1}, \ldots, X_{n}\right)$ is such a system, then $\mathfrak{g}$ is spanned by every basic system $B\left(t \cdot X_{1}, \ldots, t \cdot X_{n}\right)$, where $t$ lies in a subset of $\mathbf{R}$ with discrete complement.

Example 1.7. (i) Let $\mathfrak{g}=\operatorname{sl}(2, \mathbf{R})$ and write $P \stackrel{\text { def }}{=}\left(\begin{array}{ll}0 & 1 \\ 0 & 0\end{array}\right), Q \stackrel{\text { def }}{=}\left(\begin{array}{ll}0 & 0 \\ 1 & 0\end{array}\right), H \stackrel{\text { def }}{=}$ $\left(\begin{array}{cc}1 & 0 \\ 0 & -1\end{array}\right)$. Then the elements $H$ and $Z \stackrel{\text { def }}{=} P+Q$ generate $\mathfrak{g}$ as a Lie algebra; the basic system $B(t \cdot H, t \cdot Z, t \cdot H)=\left\{t \cdot H, t \cdot e^{\operatorname{ad} t \cdot H} Z, t \cdot e^{\text {ad } t \cdot H} e^{\text {ad } t \cdot Z} H\right\}$ is a vector space basis of $\mathfrak{g}$ for every $t \in \mathbf{R} \backslash\{0\}$.

(ii) Let $\mathfrak{g}$ be the three-dimensional Lie algebra spanned by three vectors $X$, $Y, Z$ satisfying the relations $[X, Y]=Z,[X, Z]=-Y$ and $[Y, Z]=0$ (so that $\mathfrak{g}$ is isomorphic with the Lie algebra of the group of Euclidean motions in the plane). Then the vectors $X, Y$ generate $\mathfrak{g}$ as a Lie algebra. The basic system $B(t \cdot Y, t \cdot X, t \cdot Y)=\{t \cdot Y, t \cdot(X-t \cdot Z), t \cdot(\cos t \cdot Y+\sin t \cdot Z)\}$ is a vector space basis of $\mathfrak{g}$ if and only if $t$ is not an integer multiple of $\pi$.

We remark in passing that it is not known whether the elements $X_{1}, \ldots, X_{n}$ can be chosen so that the associated basic systems $\left.\left.B\left(t \cdot X_{1}, \ldots, t \cdot X_{n}\right), t \in\right] 0,1\right]$ are vector space bases of $\mathfrak{g}$. Note also that our arguments do not give an explicit construction of a basic system (say, by recursion). Indeed, the proof looks for basic systems of maximal dimension rather than for maximal basic systems. It is not clear that every basic system is contained in a basic system of maximal dimension. 


\section{A DIRECT PATH INTO THE INTERIOR}

We now formulate and prove the main result.

Theorem 2.1. Let $S$ be a subsemigroup of a Lie group $G$ satisfying the following condition $(\mathrm{g})$ :

(g) There is a subset $E \subseteq \mathfrak{g}$ such that $\exp \mathbf{R}^{+} \cdot E \subseteq S$ and $E$ generates $\mathfrak{g}$ as Lie algebra. Then there exist elements $X_{1}, \ldots, X_{n} \in \mathbf{R}^{+} \cdot E$ such that the analytic curve $\varphi: \mathbf{R} \rightarrow G$ given by $\varphi(t)=\exp t X_{n} \cdots \exp t X_{1}$ satisfies $\varphi(0)=\mathbf{1}$ and $\varphi(t) \in$ int $S$ for all $t \in] 0,1]$.

Proof. According to Theorem 1.1 we select $X_{1}, \ldots, X_{n}$ in $\mathbf{R}^{+} \cdot E$ in such a fashion that for all $t \in] 0,1]$, the basic system $B\left(t \cdot X_{1}, \ldots, t \cdot X_{n}\right)$ spans $\mathfrak{g}$. We shall prove that the theorem holds for these vectors.

First we show that for each $t \in] 0,1]$ the set $S$ is a neighborhood of $\varphi(t)$. For this purpose we consider the function $f:[0,1]^{n} \rightarrow G$ given by

$$
f\left(t_{1}, \ldots, t_{n}\right)=\exp t_{n} \cdot X_{n} \cdots \exp t_{1} \cdot X_{1} \text {. }
$$

It suffices to verify that the derivative

$$
d f\left(s_{1}, \ldots, s_{n}\right): \mathbf{R}^{n} \rightarrow T_{g}(G), \quad g=\exp s_{n} \cdot X_{n} \cdots \exp s_{1} \cdot X_{1}
$$

is surjective for all $\left(s_{1}, \ldots, s_{n}\right)=(t, \ldots, t)$ with $0<t \leq 1$. To carry out the calculation we identify $T_{g}(G)$ with $\mathfrak{g}$ via the isomorphism $d \rho_{g}(\mathbf{1}): \mathfrak{g}=$ $T_{1}(G) \rightarrow T_{g}(G)$, where $\rho_{g}(x)=x g$.

For $k=1, \ldots, n$ we introduce small numbers $h_{k}$ and set $t_{k} \stackrel{\text { def }}{=} s_{k}+h_{k}$. Then, with $g_{k} \stackrel{\text { def }}{=} \exp s_{k} \cdot X_{k}$,

$$
\begin{aligned}
\exp t_{n} & \cdot X_{n} \cdots \exp t_{1} \cdot X_{1}=\left(\exp h_{n} \cdot X_{n}\right) g_{n} \cdots\left(\exp h_{1} \cdot X_{1}\right) g_{1} \\
= & \left(\exp h_{n} \cdot X_{n}\right)\left(\exp h_{n-1} \cdot \operatorname{Ad}\left(g_{n}\right) X_{n-1}\right) \\
& \cdots\left(\exp h_{n} \cdot \operatorname{Ad}\left(g_{n}\right) \cdots \operatorname{Ad}\left(g_{2}\right) X_{1}\right) g_{n} \cdots g_{2} g_{1} \\
= & \left(\exp \left(h_{n} \cdot X_{n} * h_{n-1} \cdot \operatorname{Ad}\left(g_{n}\right) X_{n-1} * \cdots * h_{n} \cdot \operatorname{Ad}\left(g_{n}\right) \cdots \operatorname{Ad}\left(g_{2}\right) X_{1}\right)\right) g .
\end{aligned}
$$

This implies for every $\left(u_{1}, \ldots, u_{n}\right) \in \mathbf{R}^{n}$

$$
\begin{aligned}
& d f\left(s_{1}, \ldots, s_{n}\right)\left(u_{1}, \ldots, u_{n}\right) \\
& \quad=u_{n} \cdot X_{n}+u_{n-1} \cdot \operatorname{Ad}\left(g_{n}\right) X_{n-1}+\cdots+u_{1} \cdot \operatorname{Ad}\left(g_{n}\right) \cdots \operatorname{Ad}\left(g_{2}\right) X_{1} .
\end{aligned}
$$

Thus $d f\left(s_{1}, \ldots, s_{n}\right)$ is surjective if and only if the set

$$
C=\left\{X_{n}, \operatorname{Ad}\left(g_{n}\right) X_{n-1}, \ldots, \operatorname{Ad}\left(g_{n}\right) \cdots \operatorname{Ad}\left(g_{2}\right) X_{1}\right\}
$$

spans $\mathfrak{g}$. Now we recall that $g_{k}=\exp s_{k} \cdot X_{k}$, consequently, $\operatorname{Ad}\left(g_{k}\right)=e^{s_{k} \cdot \operatorname{ad} X_{k}}$. Therefore $C=B\left(s_{1} \cdot X_{1}, \ldots, s_{n} \cdot X_{n}\right)$. Hence by our choice of $X_{1}, \ldots, X_{n}$ and Theorem 1.1 the set $C$ spans $\mathfrak{g}$ whenever $\left.\left.s_{1}=\cdots=s_{n}=t \in\right] 0,1\right]$. We have shown that $\varphi(t) \in \operatorname{int} S$ for $t \in] 0,1]$.

Remark. If $S$ has nonempty interior then by Proposition V.0.15 of [1] we know that int $S=\operatorname{int} \bar{S}$. Thus for semigroups $S$ with int $S \neq \varnothing$, we may replace the condition " $\exp \mathbf{R}^{+} \cdot E \subseteq S$ " in 2.1 by the slightly weaker condition " $\exp \mathbf{R}^{+} \cdot E \subseteq \bar{S} "$. 
Example 2.2 ("The Parking Ramp"). Let $G$ be the semidirect product $G=$ $\mathbf{C} \rtimes \mathbf{R}$ with the multiplication $(c, t)\left(c^{\prime}, t^{\prime}\right)=\left(c+e^{i t} c^{\prime}, t+t^{\prime}\right)$. We claim that the set $S=S_{1} \cup S_{2}$, with

$$
\begin{aligned}
& S_{1} \stackrel{\text { def }}{=}\left\{\left(r e^{i s}, t\right) \mid r \in \mathbf{R}^{+}, 0 \leq s \leq t \leq \pi\right\}, \\
& S_{2} \stackrel{\text { def }}{=}\{(c, t) \mid c \in \mathbf{C}, t>\pi\},
\end{aligned}
$$

is the ray subsemigroup of $G$ which is generated by the one-parameter semigroups $\left\{(r, 0) \mid r \in \mathbf{R}^{+}\right\}$and $\left\{(0, t) \mid t \in \mathbf{R}^{+}\right\}$. (Note that $S$, though generated by only two one-parameter subsemigroups, has dense interior but is not closed in $G$.)

Let us first show that $S$ is a semigroup. Clearly, $S S_{2} \cup S_{2} S \subseteq S_{2}$. Assume that $a=\left(r_{1} e^{i s_{1}}, t_{1}\right)$ and $b=\left(r_{2} e^{i s_{2}}, t_{2}\right)$ are in $S_{1}$. If $t_{1}+t_{2}>\pi$ then $a b \in S_{2}$, so let us suppose that $t_{1}+t_{2} \leq \pi$. Then the angle between $r_{1} e^{i s_{1}}$ and $r_{2} e^{i\left(t_{1}+t_{2}\right)}$ is less than $\pi$, hence $\arg \left(r_{1} e^{i s_{1}}+r_{2} e^{i\left(s_{1}+s_{2}\right)}\right) \leq t_{1}+t_{2}$ and therefore $a b=\left(r_{1} e^{i s_{1}}+r_{2} e^{i\left(t_{1}+s_{2}\right)}, t_{1}+t_{2}\right) \in S_{1}$. Thus $S$ is a semigroup.

It remains to show that $S$ is contained in the semigroup $S_{*}$ generated by the elements $(r, 0)$ and $(0, t)$, with $r \in \mathbf{R}^{+}, t \in \mathbf{R}^{+}$. For a proof of this claim we first observe that every element $x \in G$ has a unique representation $x=\left(r e^{i s}, t\right)$, with $r \in \mathbf{R}_{+}, 0 \leq s<2 \pi, t \in \mathbf{R}$. Suppose that $x \in S$. If $s \leq t$, then $x=(0, s)(r, 0)(0, t-s) \in S_{*}$, in particular, $S_{1} \subseteq S_{*}$. Thus we are left with the task to show $x \in S_{*}$ for those $x \in S_{2}$ where $s>t$, so assume that $2 \pi>s>t>\pi$. Pick a number $\left.t^{\prime} \in\right] \pi, t\left[\right.$. Then $-\pi<t^{\prime}-2 \pi<t^{\prime}-s<$ $t^{\prime}-t<0$, hence the numbers

$$
a=\frac{r \sin s}{\sin t^{\prime}}, \quad \text { and } b=\frac{r \sin \left(t^{\prime}-s\right)}{\sin t^{\prime}}
$$

are positive. A straightforward calculation now shows that

$$
x=(b, 0)\left(0, t^{\prime}\right)(a, 0)\left(0, t-t^{\prime}\right)
$$

and we conclude that $x \in S_{*}$.

We note that the Lie algebra $\mathfrak{g}$ of $G$ can be identified with that in Example $1.7(\mathrm{ii})$, so that $\exp t \cdot Z=(t, 0), \exp t \cdot Y=(i t, 0)$ and $\exp t \cdot X=(0, t)$. The generalized one-parameter subgroup

$\varphi: \mathbf{R} \rightarrow G, \quad t \mapsto \exp t \cdot Y \exp t \cdot X \exp t \cdot Y=(i t, 0)(0, t)(i t, 0)=\left(2 t \cos \frac{t}{2} e^{i \frac{t}{2}}, t\right)$ satisfies $\varphi(0)=1$ and $\varphi(t) \in$ int $S$ for all $t>0$. If we apply the arguments in the proof of Theorem 2.1 to this example then we only get $\varphi(t) \in$ int $S$ for all $t$ outside a discrete set (cf. Example 1.7(ii)). It is still open whether a generalized one-parameter subsemigroup of $\bar{S}$ can intersect the boundary in a nonsingleton discrete subset.

The proof of the following local version of the preceding theorem is left to the reader. For the definition of a local semigroup with respect to some Campbell-Hausdorff neighborhood in a Lie algebra, see [1, IV.1.2]. 
Remark 2.3. Let $N$ be a C-H neighborhood in $\mathfrak{g}$ and $S \subseteq N$ a local subsemigroup with respect to $N$. Suppose that there is a set $E \subseteq S$ with $[0,1] \cdot E=E$ generating $\mathfrak{g}$ as a Lie algebra. Then there exists an analytic curve $\varphi:[0,1] \rightarrow \mathfrak{g}$ with $\varphi(0)=0$ and $\varphi(t) \in$ int $S$ for all $t \in] 0,1]$.

Corollary 2.4. Under the hypotheses of Theorem 2.1, there is an analytic curve $\sigma: \mathbf{R} \rightarrow G$ such that

(i) $\sigma(0)=\mathbf{1}$,

(ii) $\sigma(\mathbf{R} \backslash\{0\}) \subseteq$ int $S$,

(iii) $\dot{\sigma}(0)=0$.

Proof. We define $\alpha: \mathbf{R} \rightarrow \mathbf{R}$ by setting $\alpha(t)=\left(\frac{2}{\pi} \arctan t\right)^{2}$. Then $\alpha$ is analytic, $\dot{\alpha}(0)=0$, and $0 \leq \alpha(t)<1$. Moreover, $\alpha(t)=0$ if and only if $t=0$. Now let $\varphi: \mathbf{R} \rightarrow G$ be as in Theorem 2.1. We set $\sigma=\varphi \circ \alpha$ and observe that all requirements are satisfied.

Corollary 2.5 (The Analytic Deformation Theorem). Let $S$ denote a subsemigroup of a Lie group $G$ satisfying condition (g) of Theorem 2.1. Suppose that $\gamma: I \rightarrow G$ denotes an analytic curve defined on an interval $I \subseteq \mathbf{R}$ taking its values in int $S$. Then there is an analytic function $\Gamma: I \times R \rightarrow G$ with $0 \in I$ such that

(i) $\Gamma(t, 0)=\gamma(t)$ for all $t \in I$,

(ii) $\Gamma((I \backslash\{0\}) \times(\mathbf{R} \backslash\{0\})) \subseteq$ int $S$,

(iii) $\Gamma(0, r)=\gamma(0)$ for all $r \in \mathbf{R}$,

(iv) $\left.\frac{d}{d t}\right|_{t=0} \Gamma(t, r)=\dot{\gamma}(0)$ for every $r \in \mathbf{R}$.

Proof. We take the function $\sigma: \mathbf{R} \rightarrow G$ of Corollary 2.4 and define $\Gamma(t, r)=$ $\sigma(r t) \gamma(t)$. Then $\Gamma(t, 0)=\alpha(0) \gamma(t)=\gamma(t)$. This proves (i). Since int $S$ is an ideal of $\overline{\operatorname{int} S}$ by [1, Proposition V.0.15], we conclude (ii) from the fact that $\sigma(r t) \in$ int $S$ for $t \neq 0$. Next $\Gamma(0, r)=\sigma(0) \gamma(0)=\gamma(0)$, proving (iii). Finally $\left.\frac{d}{d t}\right|_{t=0} \Gamma(r, t)=\left.\frac{d}{d t}\right|_{t=0} \sigma(r t) \gamma(t)=r \cdot \dot{\sigma}(r t)+\dot{\gamma}(0)=\dot{\gamma}(0)$ in view of Corollary 2.4(iii). (We note that this calculation takes place in the tangent space $T_{\gamma(0)}(G)$ which is identified with $\mathfrak{g}$ via $d \rho_{\gamma(0)}(\mathbf{1})$.)

If, in the preceding corollary, $\gamma$ is only assumed to be a smooth curve, then $\Gamma$ retains all of its properties except that, in general, it is only smooth but no longer analytic.

For the following corollary we need a lemma:

Lemma 2.6. Let $T$ be an open subsemigroup of a Lie group $G$ and suppose that $T$ has a connected component $T_{0}$ with $1 \in \overline{T_{0}}$. Then the closure of any connected component of $T$ is open in $\bar{T}$. Thus the connected components of $\bar{T}$ are just the closures of the connected components of $T$.

Proof. Let $p \in \bar{T}$. If $q$ lies in the open neighborhood $T_{0}^{-1} T_{0} p \cap \bar{T}$ of $p$ in $\bar{T}$ then the connected subsets $T_{0} q$ and $T_{0} p$ of $\operatorname{int}(\bar{T})=T$ have nonempty 
intersection, hence belong to the same connected of $T$. Thus $p$ and $q$ lie in the same connected component of $\bar{T}$ and the assertion follows.

A semigroup $S$ in a Lie group satisfying condition $(\mathrm{g})$ of Theorem 2.1. is certainly preanalytic since $S \cup S^{-1}$ generates $G$. Its tangent Lie wedge $\mathbf{L}(S)$ is therefore well defined as the set of all subtangent vectors of the set $\exp ^{-1}(S)$ in $\mathfrak{g}$ at 0 . (Cf. [1].)

Corollary 2.7. Suppose that $S$ is a subsemigroup of a Lie group $G$ satisfying condition $(\mathrm{g})$ of Theorem 2.1. Then the following conclusions hold.

(i) For each vector $X \in \mathbf{L}(S)$ there is an analytic curve $\varphi: \mathbf{R} \rightarrow G$ such that $\varphi(0)=\mathbf{1}, \dot{\varphi}(0)=X$ and $\varphi(t) \in \operatorname{int} S$ for all $t>0$.

(ii) For each vector $X$ in the algebraic interior algint $\mathbf{L}(S)$, there is a generalized one-parameter subgroup

$$
\sigma: \mathbf{R} \rightarrow G, \quad t \mapsto \exp t \cdot X_{1} \exp t \cdot X_{2} \cdots \exp t \cdot X_{n},
$$

such that $\sigma(0)=\mathbf{1}, \dot{\sigma}(0)=X$ and $\sigma(t) \in \operatorname{int} S$ for every $t \in] 0,1]$.

(iii) For each $s \in \overline{\langle\exp \mathbf{L}(S)\rangle}$, there is a piecewise analytic curve $\psi:[0,1] \rightarrow$ $S$ such that $\psi(0)=1, \psi(1)=s$ and $\psi(] 0,1[) \subseteq$ int $S$. In particular, $\overline{\langle\exp \mathbf{L}(S)\rangle}$ is connected by smooth curves.

(iv) If $a$ and $b$ are two points in the closure of a path component $C$ of $\bar{S}$, then there is a smooth curve $\gamma:[0,1] \rightarrow \bar{C}$ with $\gamma(0)=a, \gamma(1)=b$, and $\gamma(] 0,1[) \subseteq \operatorname{int} C$. As a consequence, $C$ is open-closed in $\bar{S}$ and connected by smooth arcs.

Proof. For a proof of (i) we set $I=[0, \infty[$ and define $\gamma: I \rightarrow G$ by $\gamma(t)=$ $\exp t \cdot X$. Then $\gamma(t) \in \overline{\operatorname{int} S}$ for all $t \in I$ by [1, Theorem V.1.13], and $\dot{\gamma}(0)=X$. Applying Corollary 2.4 and setting $\varphi(t)=\Gamma(t, 1)$ we obtain (i).

(ii) We know by Theorem 2.1 that there is a generalized one-parameter subgroup $\sigma_{0}: \mathbf{R} \rightarrow G, t \mapsto \exp t \cdot X_{1} \exp t \cdot X_{2} \cdots \exp t \cdot X_{n}$, such that $\sigma_{0}(0)=\mathbf{1}$ and $\sigma_{0}(t) \in \operatorname{int} S$ for every $\left.\left.t \in\right] 0,1\right]$. Write $Y$ for the velocity $X_{1}+\cdots+X_{n}$ of $\sigma_{0}$ at 0 . Since $X \in$ algint $\mathbf{L}(S)$ there is an $\varepsilon>0$ such that $X-\varepsilon \cdot Y \in \mathbf{L}(S)$. Then the generalized one-parameter group $\sigma: \mathbf{R} \rightarrow G, t \mapsto \exp t \cdot(X-\varepsilon \cdot Y) \sigma_{0}\left(t_{\varepsilon}\right)$ has the required properties.

In order to prove assertion (iii) we consider any $s \in \overline{\langle\exp \mathbf{L}(S)\rangle}$. Then $t \mapsto$ $s \varphi(t)$ is an analytic curve connecting $s$ with $s_{1}=s \varphi(1)$, and $\left.s \varphi(] 0,1\right] \subseteq$ $\operatorname{int}\langle\exp \mathbf{L}(S)\rangle \subseteq \operatorname{int} S$ since $\operatorname{int}\langle\exp \mathbf{L}(S)\rangle$ is an ideal of $\overline{\langle\exp \mathbf{L}(S)\rangle}$. Further, $s \mapsto \varphi(s):[0,1] \rightarrow G$ is an analytic curve with $\varphi(] 0,1]) \subseteq \operatorname{int}\langle\exp \mathbf{L}(S)\rangle$. The two points $s_{1}$ and $s_{2}=\varphi(1)$ may be connected in the path connected open submanifold $\operatorname{int}\langle\exp \mathbf{L}(S)\rangle$ by a piecewise analytic arc. Joining these three curves with appropriate parametrization, we obtain $\psi$. Finally, with the aid of $C^{\infty}$-smoothing of the corners we find a smooth curve joining 1 and $s$ through the interior of $S$.

(iv) Let $\varphi:[0,1] \rightarrow S$ denote a smooth path with $\varphi(0)=1$ and $\varphi(10,1]) \subseteq$ int $S$. Then $C \varphi([0,1])$ is a path connected set meeting $C$, hence is in $C$. 
First let us assume that $a$ and $b$ are in $C$. If $\gamma: I \rightarrow \bar{S}$ is any path connecting $a$ and $b$ then $t \mapsto \gamma(t) \varphi(1)$ is a path connecting $a^{\prime}=a \varphi(1)$ with $b^{\prime}=b \varphi(1)$ through the interior of $S$. Thus the points $a^{\prime}$ and $b^{\prime}$ are in the same path component of the manifold int $S$, hence they can be connected by a smooth path in the interior of $S$. But $t \mapsto a \varphi(t), b \varphi(t)$ are analytic curves connecting $a$ with $a^{\prime}$ with $b$ with $b^{\prime}$ through the interior of $S$, respectively. We find a smooth path connecting $a$ with $b$ in $\bar{S}$ running in int $S$-with the possible exception of the endpoints-by connecting the arcs we found and by smoothing corners.

This shows that $C$ has dense interior and that its interior int $C$ is path connected. Now assume that $a$ is in $\bar{C}$. If $S_{1}$ denotes the arc component of 1 , then $C S_{1}$ is arc connected and contains $C$, hence $C S_{1} \subseteq C$. Then $\overline{C S_{1}} \subseteq \bar{C}$, and thus $a S_{1}$ is an arc connected subset of $\bar{C}$ containing the open set $a\left(\right.$ int $\left.S_{1}\right)$. Since int $C$ is dense in $C$, we know that $a S_{1} \cap C \neq \varnothing$, and this shows $a \in C$ since $C$ is a maximal arcwise connected subset. It follows that $C$ is closed.

Now we apply Lemma 2.6 with the dense ideal $T=\operatorname{int} S$ of $S$ and consider $c \in \operatorname{int} C \subseteq T$. Then the component $T_{c}$ is a manifold, hence is path connected and thus $T_{c} \subseteq C$. Now Lemma 2.6 shows that $\bar{T}_{c}$ is the connected component of $c$ in $\bar{T}=\bar{S}$ whence $C \subseteq \overline{T_{c}}$, that is, $\bar{C}=\bar{T}_{c}$. Since Lemma 2.6 also shows that $\bar{T}_{c}$ is open in $\bar{S}$, we know that $\bar{C}$ is open in $\bar{S}$. Now (iv) is completely proved.

Example 0.2 shows that the assertion of (ii) cannot be improved.

In this entire section we explicitly or implicitly made the hypothesis that the Lie algebra $\mathfrak{g}$ is generated by $\mathbf{L}(S)$. This hypothesis is necessary in view of [1, Proposition V.2.1 and Theorem V.1.13]. However, if $S$ is an an arbitrary infinitesimally generated subsemigroup of a Lie group group $G$ (see [1, Definition V.1.11]), then the results of this section apply still to the Lie group $G(S)=\langle\exp \mathbf{L}(S) \cup \exp -\mathbf{L}(S)\rangle$ and the subsemigroup $S$ with the induced topology. The injective morphism $l: G(S) \rightarrow G$ implemented by the inclusion is an immersion of analytic manifolds. Thus any analytic curve $\gamma: I \rightarrow G(S)$ produces an analytic curve $l \circ \gamma: I \rightarrow G$. Hence the results of Corollary 2.6(i) and the relation between ray semigroups and infinitesimally generated semigroups expressed in Theorems V.1.13 and V.1.16 of [1] yield the following results:

Corollary 2.8. Let $S$ denote an infinitesimally generated subsemigroup of the Lie group $G$. Then for each $X \in \mathbf{L}(S)$ there is an analytic curve $\psi: \mathbf{R} \rightarrow G$ such that $\psi(0)=\mathbf{1}, \dot{\psi}(0)=X$, and $\psi(] 0, \infty[) \subseteq \operatorname{int}_{G(S)} S$.

The Analytic Deformation Theorem for infinitesimally generated semigroups has a simple generalization to this setting. Its formulation is an exercise. 


\section{REFERENCES}

1. J. Hilbert, K. H. Hofmann, and J. D. Lawson, Lie groups, convex cones, and semigroups, Oxford Univ. Press, 1989.

2. K. H. Hofmann and W. A. F. Ruppert, On foliations induced by congruences, Monatsh. Math. 106 (1988), 179-204.

3. W. A. F. Ruppert, On open subsemigroups of Lie groups, Semigroup Forum 39 (1989), 347-362 (to appear).

Fachbereich Mathematik, Technische Hochschule Darmstadt, Schlossgartenstrasse 7, D-6100 DARMSTADT, FR Germany (XMATDA4L @ DDATHD21.BITNET)

InSTITUT FÜr MATHEMATIK UND ANGEWANDTE STATISTIK, UNIVERSitÄt FÜr BodenKUltUR, Gregor Mendelstrasse 33, A-1180 Wien, Austria (H506T0@ AWIBOK01.BitNet) 